\title{
A INCIDÊNCIA DE DENGUE ASSOCIADA A FATORES SOCIOECONÔMICOS NO MUNICÍPIO DE CAMPOS DOS GOYTACAZES - RJ
}

\author{
Gabriela Arêdes Lima1 \\ Bianca Rocha Araújo ${ }^{2}$ \\ Mateus Vargas Peres ${ }^{3}$ \\ Thais Helena Avemedio ${ }^{4}$ \\ Rafael Guerra ${ }^{5}$ \\ Anderson Ribeiro ${ }^{6}$ \\ Adriano Rodrigues de Paula ${ }^{7}$ \\ César Ronald Pereira ${ }^{8}$ \\ Richard lan Samuels ${ }^{9}$
}

Resumo: A dengue é considerada pela Organização Mundial de Saúde (OMS) uma arbovirose de maior relevância no mundo. O agente etiológico da doença é um vírus do gênero Flavivírus, pertencente à família Flaviridae e é classificado em quatro sorotipos (DENV 1-4). O mosquito vetor, Aedes aegypti, apresenta-se em grande parte do território nacional e isso se deve à urbanização acelerada, sem planejamento, característica dos centros urbanos de países em desenvolvimento. Sem vacina contra a dengue, o controle da população de A. aegypti torna-se importante. Levantamentos de incidência de dengue também podem direcionar os programas de controle do vetor para locais com maiores casos suspeitos e confirmados. $O$ presente trabalho realizou um levantamento de incidência da doença dengue em 6 bairros de Campos dos Goytacazes, Estado do Rio de Janeiro, Brasil. Observou que os bairros com melhor infraestrutura e população com melhor renda a incidência de dengue foi significativamente baixa, comparado com bairros de periferia.

Palavras-chave: Dengue; Fatores socioeconômicos; Vigilância epidemiológica.

\footnotetext{
${ }^{1}$ Faculdade de Medicina de Campos - Setor de Epidemiologia e Saúde Coletiva, Av. Alberto Torres, 217, Campos dos Goytacazes-RJ, Brasil. E-mail: gabi_aredes@hotmail.com.

${ }_{2}^{2}$ Faculdade de Medicina de Campos - Setor de Epidemiologia e Saúde Coletiva, Av. Alberto Torres, 217, Campos dos Goytacazes-RJ, Brasil. E-mail: bianca-araujoo@hotmail.com.

${ }^{3}$ Faculdade de Medicina de Campos - Setor de Epidemiologia e Saúde Coletiva, Av. Alberto Torres, 217, Campos dos Goytacazes-RJ, Brasil. E-mail: matperesvargas@gmail.com.

${ }^{4}$ Faculdade de Medicina de Campos - Setor de Epidemiologia e Saúde Coletiva, Av. Alberto Torres, 217, Campos dos Goytacazes-RJ, Brasil. E-mail: thaishelenajf@gmail.com

${ }^{5}$ Faculdade de Medicina de Campos - Setor de Epidemiologia e Saúd.e Coletiva, Av. Alberto Torres, 217, Campos dos Goytacazes-RJ, Brasil. E-mail: afaelviannab@yahoo.com.br.

${ }^{6}$ Universidade Estadual do Norte Fluminense - Centro de Ciências e Tecnologias Agropecuárias - Laboratório de Entomologia e Fitopatologia, Av. Alberto Lamego, 2000, Campos dos Goytacazes-RJ, Brasil. E-mail: anderson.ribeirorj@yahoo.com.br.

${ }^{7}$ Universidade Estadual do Norte Fluminense - Centro de Ciências e Tecnologias Agropecuárias - Laboratório de Entomologia e Fitopatologia, Av. Alberto Lamego, 2000, Campos dos Goytacazes-RJ, Brasil. E-mail: biodepaula@yahoo.com.br.

${ }^{8}$ Faculdade de Medicina de Campos - Setor de Epidemiologia e Saúde Coletiva, Av. Alberto Torres, 217, Campos dos Goytacazes-RJ, Brasil. E-mail: cesaronald@bol.com.br.

${ }^{9}$ Universidade Estadual do Norte Fluminense - Centro de Ciências e Tecnologias Agropecuárias - Laboratório de Entomologia e Fitopatologia, Av. Alberto Lamego, 2000, Campos dos Goytacazes-RJ, Brasil. E-mail: richard@uenf.br.
} 